\title{
Contribuição do Pró-Saúde para a reorientação da formação em Odontologia e implementação das Diretrizes Curriculares Nacionais: estudo de casos
}

\author{
Rosa Maria Eid Weiler*; Maria Sylvia de Souza Vitalle**; Ana Estela Haddad***; Fabian Calixto \\ Fraiz****; Maria Sueli Marques Soares*****; Emílio Carlos Sponchiado Júnior******; Lilian de \\ Fátima Guedes Amorim*******; Maria Ercília de Araújo $* * * * * * * *$; Ana Maria Gondim \\ Valença**********
}

* Prof. Externo do Curso de Especialização em Adolescência para Equipe Multidisciplinar do Departamento de Pediatria da Universidade Federal de São Paulo, UNIFESP

** Prof. Adjunto do Setor de Medicina do Adolescente, Universidade Federal de São Paulo, UNIFESP

Prof. Associado do Departamento de Ortodontia e Odontopediatria da Faculdade de Odontologia da Universidade de São Paulo, USP

**** Prof. Associado da Universidade Federal do Paraná, UFPR Prof. Associado da Universidade Federal da Paraíba, UFPB

******* Prof. Adjunto da Universidade Federal do Amazonas, UFAM

******* Professora Assistente de Odontopediatria da Faculdade de Odontologia da Universidade Federal de Goiás, UFG

******** Prof. Associado Livre Docente em Saúde Coletiva, Universidade de São Paulo, USP

Prof. Titular da Universidade Federal da Paraíba, UFPB

Recebido em 30/11/2016. Aprovado em 30/03/2017.

\begin{abstract}
RESUMO
O Pró-Saúde é uma iniciativa do Ministério da Saúde com a parceria do Ministério da Educação, de amplo alcance e capilaridade, instituído e implementado a partir de 2006 e voltado para a reorientação da formação profissional em Saúde. O objetivo do presente estudo foi verificar de que forma e com quais estratégias estão sendo conduzidas as mudanças curriculares em cursos de graduação de Odontologia, em universidades públicas brasileiras, inscritas neste programa. Trata-se de um estudo de casos. Foram selecionados cinco cursos de graduação, um em cada macrorregião do país, e aplicados questionários de percepção aos coordenadores ou docente representante de cada curso, e a um grupo de estudantes, sobre as mudanças em processo. Os resultados apontaram que o Pró-Saúde tem sido um efetivo instrumento indutor de mudanças na direção da implementação das Diretrizes Curriculares Nacionais (DCN). Os estágios extramuros apresentam grande variação no seu planejamento e representam uma ferramenta importante para o desenvolvimento das competências requeridas pelas DCN. Em relação à abordagem pedagógica, ainda predominam práticas tradicionais baseadas na transmissão vertical de conhecimentos e a integração das disciplinas ocorre, principalmente, em seminários modulares e disciplinas profissionalizantes.

Descritores: Educação em Odontologia. Currículo. Sistema Único de Saúde. Recursos Humanos em Saúde.
\end{abstract}




\section{INTRODUÇÃO}

As Diretrizes Curriculares Nacionais (DCN) dos cursos de graduação em Odontologia vigentes desde $2002^{1}$ devem orientar o planejamento do projeto político pedagógico e o currículo dos cursos, aliado ao Plano de Desenvolvimento Institucional (PDI), que responde por especificidades de cada Instituição de Ensino Superior, e sua inserção na comunidade local.

No contexto da educação superior, a flexibilização preconizada pelas DCN confere às Instituições de Ensino Superior (IES) novos graus de liberdade que possibilitam o desenho de currículos inovadores, adequados à realidade regional e às respectivas vocações de cada curso. A substituição do currículo mínimo pela formação baseada em competências representa um avanço e contribui para a integração ensinoserviço, para a aplicação de metodologias ativas no processo de ensino-aprendizagem e para a efetivação do princípio da relevância social da ação acadêmica. Na área da Saúde, as DCNs reforçam a necessidade de considerar a formação profissional de acordo com o modelo de atenção à saúde preconizado pelo Sistema Único de Saúde (SUS) ${ }^{2}$.

Estudos desenvolvidos sobre a avaliação da aplicação das DCN nos cursos de Odontologia verificaram que as concepções de ensinoaprendizagem oscilavam entre modelos tradicionais e inovadores ${ }^{3}$; que experiências positivas na aproximação dos cursos de graduação com os serviços públicos de saúde podem ser alcançadas por meio de uma configuração satisfatória dos estágios extramuros $^{4}$; e que existe a dificuldade dos professores se adaptarem a um currículo de integração, devido a uma formação docente prévia ao advento do SUS e construída sobre a fragmentação de conteúdo 5 .

O Ministério da Saúde (MS), por meio da
Secretaria de Gestão do Trabalho e da Educação na Saúde (SGTES), e o Ministério da Educação (MEC), por meio da Secretaria de Educação Superior (Sesu) e do Instituto Nacional de Estudos e Pesquisas Educacionais Anísio Teixeira (INEP), formularam e desenvolveram as políticas nacionais de educação superior e formação dos profissionais da saúde em progressiva articulação e integração. Entre elas, o Programa Nacional de Reorientação Profissional na Saúde (Pró Saúde), que tem como objetivo geral induzir mudanças na formação profissional em saúde, tendo como referência as DCN, promovendo a integração ensino-serviço, a compreensão ampliada do processo saúdeadoecimento, considerando os determinantes sociais e o uso de metodologias ativas no processo de ensino-aprendizagem, com vistas à avançar na atenção à saúde da população no modelo preconizado pelo $\mathrm{SUS}^{2}$.

O Pró-Saúde promoveu a integração ensino-serviço, inicialmente envolvendo os cursos de graduação da área da saúde com as secretarias de saúde e gradualmente outros serviços e os demais níveis de atenção, buscando atuar na lógica das redes de atenção à saúde ${ }^{6}$.

Um estudo conduzido pelos Ministérios da Saúde e da Educação, pouco antes da instituição do Pró-Saúde, investigou a aderência às DCN dos cursos de graduação em Medicina, Enfermagem e Odontologia. De acordo com os resultados, embora as DCN estivessem devidamente retratadas nos projetos político pedagógicos dos cursos que foram autorizados pelo Ministério da Educação após as DCN, e que fizeram parte do estudo, a análise dos relatórios das visitas de avaliação in loco desses mesmos cursos, indicam que as DCN não foram efetivamente incorporadas no planejamento e nas práticas pedagógicas ${ }^{7}$.

O objetivo do presente estudo foi verificar de que forma e com quais estratégias estão sendo 
conduzidas as mudanças curriculares em cursos de graduação em Odontologia de universidades públicas de cada região geográfica do Brasil, inscritas no Pró Saúde.

\section{METODOLOGIA}

Trata-se de um estudo de caso, que obteve aprovação do Comitê de Ética em Pesquisa, sob parecer de número 311.503. O Termo de Consentimento Livre e Esclarecido foi assinado pelos participantes, antes de responderem ao questionário.

\section{Amostra}

A amostra foi constituída por cinco cursos de graduação em Odontologia de universidades públicas, tendo como critério a escolha de um curso de cada uma das cinco macrorregiões brasileiras, localizados em capitais. Participaram do estudo, um docente representante de cada curso e 149 estudantes do último ano dos respectivos cursos. Foram sorteados os estados de cada região e se o curso da IES pública selecionada não aceitasse participar, procedia-se novo sorteio.

Os cinco cursos de graduação em Odontologia participantes foram nomeados da seguinte forma: Curso A (CA), Curso B (CB), Curso C (CC), Curso D (CD) e Curso E (CE), sendo uma instituição pública representante de cada uma das cinco regiões do país.

Os docentes representantes dos cursos exerciam as seguintes funções dentro das IES: coordenadores de graduação $(\mathrm{CA}, \mathrm{CB}$ e $\mathrm{CD})$ e professores envolvidos com questões curriculares da graduação (CC e CE).

\section{Instrumentos e execução}

Os convites foram feitos por telefone e correio eletrônico. Os questionários foram encaminhados para os representantes docentes por correio eletrônico com dois links: um para o questionário dos docentes e outro para o questionário dos alunos. Os representantes docentes responderam ao questionário a eles destinado e encaminharam o outro link para que os alunos respondessem. $\mathrm{O}$ acesso eletrônico aos questionários era feito por meio do banco de dados do Google Docs (Google Inc, Mountain View, Califórnia, EUA).

Os questionários direcionados aos docentes representantes das IES e aos estudantes foram desenvolvidos com base nas competências requeridas pelas DCN dos cursos de Odontologia, entre as quais: a) estratégias utilizadas pelas instituições para desenvolver capacidade de formação de um profissional crítico capaz de questionar, trabalhar em equipe, considerando a realidade social, capacidade de atuar de acordo com os princípios, diretrizes e modelo de atenção à saúde do SUS e b) estratégias para desenvolver um projeto pedagógico centrado no aluno como sujeito da aprendizagem e apoiado no professor como facilitador e tutor do processo e, finalmente, c) estratégias para integração das disciplinas básicas e clínicas. O questionário dirigido aos representantes docentes apresentava 20 questões, das quais 10 eram semiabertas, 7 eram fechadas e 3 apresentavam escalas com escores ou respostas que mostravam o grau de concordância com a afirmação (escala Likert).

O questionário dos alunos apresentava também 20 questões, sendo 10 delas semiabertas, 3 fechadas e 7 apresentando escalas de concordância (Likert). A abordagem, nesse caso, teve por objetivo investigar a opinião dos estudantes sobre a efetividade das estratégias.

Foi realizado um pré-teste para validação dos questionários, procedendo-se o ajuste do conteúdo e analisando-se o padrão das respostas. O pré-teste foi aplicado a 5 estudantes e 1 docente. Os participantes não compuseram a amostra da pesquisa.

Os questionários, desenvolvidos para preenchimento online, depois de finalizados 
geraram um link (um para cada formulário) o qual foi enviado por correio eletrônico. Ao serem respondidos, cada um deles era direcionado a uma planilha específica no aplicativo utilizado.

Os questionários ficaram disponíveis para preenchimento pelo período de 12 meses, de agosto de 2013 a agosto de 2014. Durante todo o período da pesquisa e, na interpretação dos dados, os docentes, representantes de cada curso envolvido, foram consultados sempre que surgiam dúvidas, ou a necessidade de aprofundar-se em um tema que havia sido colocado pelos alunos na opção de resposta aberta.

\section{Análise Estatística}

Para as respostas dos docentes representantes dos cursos e dos estudantes foi utilizada a análise estatística descritiva dos dados. Em relação aos estudantes, foi feita a avaliação das variáveis pelo total de respostas por estudantes e por curso. Em uma segunda etapa da análise, as ocorrências classificadas como "outras" no questionário foram agrupadas por similaridade.

\section{RESULTADOS}

A estrutura dos cursos de acordo com as disciplinas eletivas e estágios extramuros é demonstrada na tabela 1.

Em relação à forma de integração das diversas disciplinas ao longo do curso, as respostas estão expressas na tabela 2 .

Os cursos que utilizam seminários por blocos de disciplinas para integrá-las, relatam que as agrupam de acordo com as disciplinas dadas em cada semestre (CD, CC. CB e CA), mas, agrupando-as ainda, devido à abordagem biológica ou social (CD, $\mathrm{CB}$ e CA).

$\mathrm{Na}$ questão sobre a exigência de trabalho de conclusão de curso (TCC), o único curso entre os cinco que não exige é o CD. Para incentivar os alunos a lerem artigos científicos de forma crítica, todos os cursos relatam que a disciplina de Metodologia Científica é a principal responsável por desenvolver essa habilidade nos alunos, além do incentivo de várias outras disciplinas no desenvolvimento de pesquisas (CE, CD, CC e CA) e do próprio TCC (CA). Essa disciplina é ofertada no início dos cursos: primeiro semestre ( $\mathrm{CA}, \mathrm{CB}$ e $\mathrm{CE}$ ), segundo semestre $(C D)$ e terceiro semestre $(C C)$.

$$
\text { Em relação às disciplinas }
$$

profissionalizantes, encontrou-se os seguintes grupos: os que possuem disciplinas individualizadas como Endodontia, Periodontia, Prótese, etc. (CE, CD e $\mathrm{CB}$ ) e os que as apresentam em módulos integrados (CA e CC).

Com relação à estratégia usada para trabalhar a relação interdisciplinar, os cursos, com exceção do $\mathrm{CE}$, citaram o estágio extramuros, no qual se trabalha em equipes com vários profissionais de saúde. $\mathrm{O} \mathrm{CE}$, apesar de ter estágios extramuros no seu currículo, não o considera uma estratégia para isso e diz que não há treinamento específico para o desenvolvimento dessa habilidade. Por outro lado, todos os cursos concordam que os estágios extramuros são a estratégia para que os estudantes possam exercer sua profissão, considerando o contexto social, entendendo-a como uma forma de participação e contribuição para a transformação e melhoria da sociedade.

O conjunto de questões que usou a escala Likert teve por objetivo avaliar a percepção dos docentes e dos estudantes quanto ao grau de utilização das metodologias ativas no processo de ensino-aprendizagem. A variação nos escores foi correspondente às seguintes afirmativas: 1 (usa apenas transmissão vertical), 2(usa muito), 3(usa medianamente), 4 (usa pouco), 5 (não usa transmissão vertical). Os escores resultantes foram: 2 (CE e CD), 3 (CC e CB) e 4 (CA).

$\mathrm{O}$ número de estudantes por curso da amostra está expresso na tabela 3. 
A tabela 4 resume as respostas mais curso eram importantes para o desenvolvimento frequentes sobre quais estratégias aplicadas no das competências preconizadas pelas DCN ${ }^{1}$.

Tabela 1 - Características dos cursos, duração dos estágios extra muros e das disciplinas eletivas

\begin{tabular}{|c|c|c|c|c|c|}
\hline & \multicolumn{4}{|c|}{ ESTRUTURA E CARACTERÍSTICAS DOS CURSOS } & \multirow[b]{2}{*}{$\mathrm{CE}$} \\
\hline & $\mathrm{CA}$ & $\mathrm{CB}$ & $\mathrm{CC}$ & $\mathrm{CD}$ & \\
\hline $\begin{array}{l}\text { Carga Horária Total } \\
\text { (hs) }\end{array}$ & 4060 & 4500 & 4496 & 5175 & 4140 \\
\hline Disciplinas eletivas & Sim & Sim & Sim & Sim & Sim \\
\hline $\begin{array}{l}\text { Carga horária das } \\
\text { disciplinas eletivas }\end{array}$ & 60 & 225 & 416 & $\begin{array}{l}\text { Não inclui } \\
\text { na carga } \\
\text { horária }\end{array}$ & 90 \\
\hline $\begin{array}{l}\text { Distribuição das } \\
\text { disciplinas eletivas } \\
\text { nos semestres }\end{array}$ & $\begin{array}{l}\text { Durante todo o } \\
\text { curso }\end{array}$ & $\begin{array}{l}\text { Durante } \\
\text { todo o } \\
\text { curso }\end{array}$ & $\begin{array}{l}\text { A partir do } \\
\text { sétimo } \\
\text { semestre }\end{array}$ & $\begin{array}{l}\text { Durante } \\
\text { todo o } \\
\text { curso }\end{array}$ & $\begin{array}{l}\text { A partir do } \\
\text { sétimo } \\
\text { semestre }\end{array}$ \\
\hline Estágios extramuros & Sim & Sim & Sim & Sim & Sim \\
\hline $\begin{array}{l}\text { Carga horária dos } \\
\text { estágios extramuros }\end{array}$ & 300 & 715 & 480 & 495 & 60 \\
\hline $\begin{array}{l}\text { Distribuição do } \\
\text { estágio extramuros } \\
\text { nos semestres }\end{array}$ & $\begin{array}{l}\text { A partir do } \\
\text { quinto } \\
\text { semestre }\end{array}$ & $\begin{array}{l}\text { Durante } \\
\text { todo o } \\
\text { curso }\end{array}$ & $\begin{array}{l}\text { A partir do } \\
\text { sétimo } \\
\text { semestre }\end{array}$ & $\begin{array}{l}\text { Durante } \\
\text { todo o } \\
\text { curso }\end{array}$ & $\begin{array}{l}\text { A partir do } \\
\text { segundo } \\
\text { semestre }\end{array}$ \\
\hline $\begin{array}{l}\text { Ano de adesão ao } \\
\text { Pró Saúde }\end{array}$ & 2008 & 2005 & 2006 & 2005 & 2012 \\
\hline
\end{tabular}

CA: curso A; CB: curso B; CC: curso C; CD: curso D; CE: curso E

Tabela 2 - Estratégias aplicadas para desenvolver as competências preconizadas pelas DCN, segundo a percepção dos Coordenadores ou Docentes representantes dos cursos de graduação

\begin{tabular}{|c|c|c|c|c|c|}
\hline & & ESTRATÉC & IAS & & \\
\hline & $\mathrm{CA}$ & $\mathrm{CB}$ & $\mathrm{CC}$ & CD & $\mathrm{CE}$ \\
\hline $\begin{array}{l}\text { Integração do } \\
\text { conteúdo ao longo de } \\
\text { todo o curso }\end{array}$ & $\begin{array}{l}\text { Disciplinas } \\
\text { profissionalizantes }\end{array}$ & $\begin{array}{l}\text { Seminários } \\
\text { integrados }\end{array}$ & $\begin{array}{l}\text { Seminários } \\
\text { integrados }\end{array}$ & $\begin{array}{l}\text { Seminários } \\
\text { integrados }\end{array}$ & $\begin{array}{l}\text { Não há } \\
\text { estratégia }\end{array}$ \\
\hline $\begin{array}{l}\text { Metodologias de } \\
\text { ensino-aprendizagem }\end{array}$ & $\begin{array}{l}\text { Seminários, } \\
\text { disciplinas, } \\
\text { profissionalizantes }\end{array}$ & $\begin{array}{l}\text { Seminários, } \\
\text { pesquisa e } \\
\text { moodle }\end{array}$ & $\begin{array}{l}\text { Seminários, júri } \\
\text { simulado, } \\
\text { dramatização }\end{array}$ & Pesquisas & Seminários \\
\hline $\begin{array}{l}\text { Integração entre ciclo } \\
\text { básico e clínico }\end{array}$ & $\begin{array}{l}\text { Disciplinas } \\
\text { Estomatologia e } \\
\text { Patologia }\end{array}$ & $\begin{array}{l}\text { Seminários } \\
\text { integrados }\end{array}$ & $\begin{array}{l}\text { Nada específico, } \\
\text { seminários. } \\
\text { Integrados }\end{array}$ & Pesquisa & Nada específico \\
\hline $\begin{array}{l}\text { Clínica de } \\
\text { especialidades x } \\
\text { Clínica integrada }\end{array}$ & $\begin{array}{l}\text { Integradas desde } \\
\text { início }\end{array}$ & Individualizadas & $\begin{array}{l}\text { Integradas desde } \\
\text { início }\end{array}$ & Individualizadas & Individualizadas \\
\hline $\begin{array}{l}\text { Trabalho em equipe } \\
\text { multidisciplinar }\end{array}$ & $\begin{array}{l}\text { Estágios } \\
\text { extramuros }\end{array}$ & $\begin{array}{l}\text { Estágios } \\
\text { extramuros }\end{array}$ & $\begin{array}{l}\text { Estágios } \\
\text { extramuros }\end{array}$ & $\begin{array}{l}\text { Estágios } \\
\text { extramuros }\end{array}$ & $\begin{array}{l}\text { Não há } \\
\text { estratégias }\end{array}$ \\
\hline $\begin{array}{l}\text { Exercer profissão de } \\
\text { forma articulada }\end{array}$ & $\begin{array}{l}\text { Estágios } \\
\text { extramuros }\end{array}$ & $\begin{array}{l}\text { Estágios } \\
\text { extramuros }\end{array}$ & $\begin{array}{l}\text { Estágios } \\
\text { extramuros }\end{array}$ & $\begin{array}{l}\text { Estágios } \\
\text { extramuros }\end{array}$ & $\begin{array}{l}\text { Estágios } \\
\text { extramuros }\end{array}$ \\
\hline
\end{tabular}


Tabela 3 - Distribuição dos estudantes por curso participante

\begin{tabular}{lccc}
\hline & Número de matriculados & Número de respondentes & Período (Semestre) \\
\hline $\mathrm{CA}$ & 32 & 25 & 10 \\
$\mathrm{CB}$ & 36 & 20 & $9-10$ \\
$\mathrm{CC}$ & 49 & 20 & $7-8$ \\
$\mathrm{CD}$ & 80 & 23 & $8-9$ \\
$\mathrm{CE}$ & 98 & 61 & $8-9$ \\
\hline Total & 295 & $149(\%)$ & \\
\hline
\end{tabular}

CA: curso A; CB: curso B; CC: curso C; CD: curso D; CE: curso E

Tabela 4 - Estratégias aplicadas para desenvolver as competências preconizadas pelas DCN, segundo a percepção dos alunos

\begin{tabular}{|c|c|c|c|c|c|}
\hline \multicolumn{6}{|c|}{ ESTRATÉGIAS } \\
\hline & CA & $\mathrm{CB}$ & $\mathrm{CC}$ & CD & CE \\
\hline $\begin{array}{l}\text { Metodologia de } \\
\text { ensino- } \\
\text { aprendizagem }\end{array}$ & $\begin{array}{l}\text { Desenvolvi- } \\
\text { mento de } \\
\text { pesquisas } \\
(64 \%)\end{array}$ & $\begin{array}{l}\text { Seminários } \\
\text { de integração } \\
(60 \%)\end{array}$ & $\begin{array}{l}\text { Seminários } \\
\text { de integração } \\
(64,7 \%)\end{array}$ & $\begin{array}{l}\text { Seminários de } \\
\text { integração } \\
(60,8 \%)\end{array}$ & $\begin{array}{l}\text { Desenvolvi- } \\
\text { mento de } \\
\text { pesquisas } \\
(42,2 \%)\end{array}$ \\
\hline $\begin{array}{l}\text { Integração entre } \\
\text { ciclo básico e } \\
\text { clínico }\end{array}$ & $\begin{array}{l}\text { Pesquisas } \\
\text { desenvolvidas } \\
(56 \%)\end{array}$ & $\begin{array}{l}\text { Seminários } \\
\text { de Integração } \\
(75 \%)\end{array}$ & $\begin{array}{l}\text { Seminários } \\
\text { de Integração } \\
(31,2 \%)\end{array}$ & $\begin{array}{l}\text { Pesquisas } \\
\text { desenvolvidas } \\
\text { Seminários de } \\
\text { integração } \\
(43,4 \%)\end{array}$ & $\begin{array}{l}\text { Pesquisas } \\
\text { desenvolvidas } \\
(60,3 \%)\end{array}$ \\
\hline $\begin{array}{l}\text { Ler artigos de } \\
\text { forma crítica }\end{array}$ & $\begin{array}{l}\text { Desenvolvi- } \\
\text { mento de } \\
\text { pesquisas } \\
(76 \%)\end{array}$ & $\begin{array}{l}\text { Desenvolvi-- } \\
\text { mento de } \\
\text { pesquisas } \\
(75 \%)\end{array}$ & $\begin{array}{l}\text { Disciplina de } \\
\text { Metodologia } \\
\text { Científica } \\
(87,5 \%)\end{array}$ & $\begin{array}{l}\text { Desenvolvi- } \\
\text { mento de } \\
\text { pesquisas } \\
(63,6 \%) \text {. }\end{array}$ & $\begin{array}{l}\text { Disciplina de } \\
\text { Metodologia } \\
\text { Científica } \\
(80,6 \%)\end{array}$ \\
\hline $\begin{array}{l}\text { Desenvolver } \\
\text { habilidade de } \\
\text { planejamento e } \\
\text { gestão }\end{array}$ & $\begin{array}{l}\text { Disciplina } \\
\text { específica para } \\
\text { isso } \\
(68 \%)\end{array}$ & $\begin{array}{l}\text { Não há } \\
\text { estratégia } \\
(50 \%)\end{array}$ & $\begin{array}{l}\text { Disciplina } \\
\text { específica } \\
\text { para isso } \\
(52,9 \%)\end{array}$ & $\begin{array}{l}\text { Disciplina } \\
\text { específica } \\
\text { para isso } \\
(95,4 \%)\end{array}$ & $\begin{array}{l}\text { Disciplina } \\
\text { específica para } \\
\text { isso } \\
(77,1 \%)\end{array}$ \\
\hline $\begin{array}{l}\text { Trabalhar em } \\
\text { equipe } \\
\text { multiprofissional }\end{array}$ & $\begin{array}{l}\text { Estágios } \\
\text { extramuros } \\
(75 \%)\end{array}$ & $\begin{array}{l}\text { Estágios } \\
\text { extramuros } \\
(85 \%)\end{array}$ & $\begin{array}{l}\text { Estágios } \\
\text { extramuros } \\
(76,4 \%)\end{array}$ & $\begin{array}{l}\text { Estágios } \\
\text { extramuros } \\
(43,4 \%)\end{array}$ & $\begin{array}{l}\text { Estágios } \\
\text { extramuros } \\
(42,1 \%)\end{array}$ \\
\hline $\begin{array}{l}\text { Exercer } \\
\text { profissão de } \\
\text { forma articulada }\end{array}$ & $\begin{array}{l}\text { Estágios } \\
\text { extramuros } \\
(96 \%)\end{array}$ & $\begin{array}{l}\text { Estágios } \\
\text { extramuros } \\
(95 \%)\end{array}$ & $\begin{array}{l}\text { Estágios } \\
\text { extramuros } \\
(82,3 \%)\end{array}$ & $\begin{array}{l}\text { Estágios } \\
\text { extramuros } \\
(59 \%)\end{array}$ & $\begin{array}{l}\text { Estágios } \\
\text { extramuros } \\
(80,7 \%)\end{array}$ \\
\hline
\end{tabular}

CA: curso A; CB: curso B; CC: curso C; CD: curso D; CE: curso E

Na questão sobre a metodologia de ensino usada para integrar o conhecimento entre o ciclo básico e clínico, nas respostas do $\mathrm{CC}$, os seminários de integração tiveram a mesma frequência que a opção "outros", que, nesse caso, citava especialmente a prática clínica e a 
aplicação das matérias básicas na clínica. No CD houve a mesma frequência de respostas $(43,4 \%)$ para seminários de integração e pesquisas desenvolvidas.

Com relação à percepção dos alunos sobre a abordagem pedagógica, na escala Likert, variando o grau do uso de metodologias de transmissão vertical a resposta por curso foi "muito" como a resposta mais frequente: $55 \%$ CB, $64,71 \%$ CC, $64 \%$ CA, $49,09 \%$ CE e $72,73 \%$ CD. No que diz respeito a quanto a transmissão do conhecimento é feita com a participação ativa do aluno, a resposta mais frequente por universidade foi: "medianamente" $(80 \% \mathrm{CB}$, $41,18 \%$ CC, $51,79 \%$ CE e $68,18 \%$ CD). A resposta mais frequente na $\mathrm{CA}$ foi "muito" $(48 \%)$.

\section{DISCUSSÃO}

Considerando as mudanças em curso em função do tempo decorrido desde o início da participação de cada um dos cursos no Pró Saúde, é possível inferir que aqueles que estão há mais tempo no programa encontram-se em estágio mais avançado no processo de reorientação da formação. Alguns cursos já estavam fazendo sua reestruturação e participar do Pró Saúde fortaleceu e ajudou a consolidar o processo, por ser este um importante indutor, embora não o único. Todos os cursos tinham professores inscritos no PET Saúde (Programa de Educação pelo Trabalho em Saúde), que inclui bolsas para docentes, preceptores, fato que adiciona indução mais acentuada à essa reestruturação. Além disso, sabe-se que o PET Saúde, instituído e implementado 2 anos após o início do Pró Saúde, foi potente em ampliar a presença do corpo docente dos cursos de graduação nos serviços de saúde, atuando mais diretamente na integração ensino-serviço, bem como também possibilitou que se avançasse no trabalho em equipe multidisciplinar desde a graduação. Este aspecto favorece o princípio da integralidade da atenção e não apenas a integração disciplinar uniprofissional. Sabe-se que uma mudança curricular em profundidade demanda tempo e pode-se considerar que o processo ainda é bem recente em todas elas, apesar das DCN serem datadas de 2002.

O Estudo de Lamers et al. ${ }^{8}$ mostra que os professores perceberam mudanças a partir da reestruturação curricular em relação aos seguintes aspectos: integração curricular, favorecimento da interdisciplinaridade, estudantes mais humanizados, metodologias de ensino que permitem espaço para mais discussão, melhorias no atendimento aos pacientes, atividades didáticas diversificadas e estágios curriculares no SUS, que, por sua vez, têm se constituído em estratégia de grande potencial para a integração da teoria com práticas .

Todos os cursos pesquisados oferecem disciplinas eletivas, o que é importante, pois confere autonomia aos estudantes e eleva não apenas a participação, mas aprimora a capacidade de aprendizado. Quando o aluno pode enfatizar o que lhe interessa sua aprendizagem se torna mais significatival ${ }^{9}$.

Estágios extramuros supervisionados, realizados na rede pública de saúde, são oferecidos em todos os cursos da amostra, conforme estabelecido pelas DCN. No entanto, os cursos adotam diferentes formatação e carga horária para os mesmos. As atividades desenvolvidas nos estágios ainda se configuram como uma questão controversa, gerando discussões sobre "onde", "como" e "quando" devem ser realizados, dentre outras competências. Os estágios curriculares são contemplados na grande maioria dos cursos de Odontologia, mas ainda se encontra num estágio de transição com relação à sua efetiva implementação conforme estabelecido pelas DCN, observando-se ainda certas divergências e 
dificuldades na sua interpretação. ${ }^{10}$.

O PET Saúde, implementado em articulação com o Pró-Saúde, como atividade de integração ensino-serviço, grupos tutoriais, onde está presente o docente (tutor) os profissionais do serviço (preceptores) e os alunos de graduação (monitores). No entanto, em alguns cursos, os alunos participam apenas de forma observacional e sem a presença de docentes. A atuação dos estudantes deve ser gradativa: observação, realização de ações educativas, visitas domiciliares e procedimentos coletivos em saúde bucal. No entanto, em alguns cursos permite-se escolher locais para estágio, que se limitam à observação. É importante que sejam realizadas avaliações periódicas para o embasamento do aprendizado reflexivo do aluno, mas isso nem sempre ocorre na prática. Problemas práticos como dificuldades de se estabelecer convênios, a capacidade instalada na rede pública de serviços, em contraponto à demanda crescente por estágio, representam ainda desafios a serem enfrentados. O CB demonstra apresentar os estágios muito bem estruturados com as Equipes de Saúde da Família. O planejamento das ações em campo é participativo e acordado com o preceptor da Unidade de Saúde da Família (USF), considerando a situação de saúde da USF e as prioridades a serem enfrentadas. Segundo a coordenação do $\mathrm{CB}$, esse processo não foi fácil no início, necessitando romper vários entraves até se chegar à situação atual. Os estágios extramuros foram escolhidos por todos os cursos, com exceção do $\mathrm{CE}$, como a melhor forma de promover o trabalho em equipe multidisciplinar e a integração entre as disciplinas e todas elas concordaram que é a melhor estratégia do curso para que os estudantes possam compreender o alcance e a dimensão da relevância social da sua atuação profissional.

Ao se perguntar para os alunos qual a estratégia do curso utilizada para integração dos conhecimentos das ciências básicas e clínicas, na opção "outros" foi muito frequente a sugestão de "discussão de casos clínicos". Observa-se também a percepção de que, atuando com as Equipes de Saúde da Família e participando efetivamente das ações, não apenas, como mero espectador, pode-se aprender muito sobre o trabalho interdisciplinar e multiprofissional. Em verdade, essa estratégia pode promover o desenvolvimento das competências gerais das DCN, comuns a todas as profissões da saúde que atuam no SUS: atenção à saúde, tomada de decisões, liderança, administração, gerenciamento, trabalho em equipe e educação permanente ${ }^{11}$.

As estratégias de integração das disciplinas mais aplicadas nos cursos pesquisados são seminários divididos por módulos de conteúdos, agrupados de acordo com as disciplinas dadas em cada semestre, ou ainda, de acordo com a maior ênfase do grupo de disciplinas, biológica ou social.

O CA considera, ainda, que a maior integração se faz nas disciplinas profissionalizantes que estão integradas nas aulas clínicas. Os estudantes do CC também consideraram a integração obtida nas disciplinas profissionalizantes tão importantes quanto a integração por seminários. Barbosa et al. ${ }^{12}$ consideram que há professores insuficientemente capacitados a atuar na metodologia ativa e de modo interdisciplinar. Ao mesmo tempo, as ações integrativas auxiliam o estudante a construir um quadro teórico-prático global mais significativo e mais próximo dos desafios presentes na realidade profissional dinâmica, complexa e única, na qual atuará como profissional ${ }^{13}$.

Quanto às disciplinas profissionalizantes, há os cursos que apresentam as clínicas divididas por disciplina, no modelo tradicional $(\mathrm{CE}, \mathrm{CD}$ e CB) e clínica integrada ao final do curso. Há, 
também, os que apresentam as clínicas totalmente integradas desde o início (CA e CC). Nesse caso, as clínicas vão sendo oferecidas durante o curso com graus de complexidade crescente, o professor orientador faz a triagem dos pacientes para corresponder às necessidades de aprendizagem de cada aluno de acordo com as metas a serem cumpridas em cada etapa Entre os alunos que tinham as clínicas integradas desde o início foi frequente relatarem que, por outro lado, não permitia o treinamento de habilidades mais complexas:

"A intenção de integrar uma habilidade com a outra não é atingida, pois em minha opinião não temos clínicas individualizadas o suficiente, dessa forma não sabemos como interagir uma especialidade com a outra".

"O currículo vigente não nos proporciona o estudo individualizado de cada disciplina, considerando a complexidade de cada uma".

Já os alunos que tinham as clínicas individualizadas, na opção "outros" citavam também as clínicas integradas, ressaltando que poderiam ser mais exploradas.

"Temos pouco tempo no semestre para realizar e treinar habilidades nas disciplinas $e$, muitas vezes, não temos como integrar o que aprendemos, deveria haver mais clínicas integradas".

Observa-se assim, que todas as escolhas implicam em perdas e ganhos, mas em se tratando da formação de um profissional, há de se encontrar uma maneira de equilibrar essa questão. A matriz da interdisciplinaridade parte das disciplinas, que não devem ser diluídas, ao contrário, devem manter suas especificidades ${ }^{14}$. Para se planejar uma clínica efetivamente integrada e formar um generalista, é preciso avançar e aprofundar a compreensão dos atuais nós críticos envolvidos no que está proposto nas DCN em oposição ao processo histórico de identidade profissional especializada e fragmentada. Nesse sentido é preciso tomar cuidado para não condenar o modelo de clínica integrada desde o início, mas avaliar se a limitação está relacionada ao fato de sua construção ainda estar impregnada de um olhar segmentado.

Ao se verificar como os cursos de graduação analisam a transmissão do conhecimento considerando-se a proporção de metodologia expositiva e de transmissão vertical em relação à metodologia que privilegia a participação dos alunos, existe uma certa divergência entre os cursos com relação ao grau de transmissão vertical no ensino, mas consideram que há grande participação dos alunos no aprendizado. No momento que se afirma que o aluno vai construir seu próprio conhecimento, ele está apenas elaborando subjetivamente acerca do que é real, a partir dos elementos, teorias e explicações que o mediador, no caso, o professor disponibiliza para ele. Por outro lado, esse conteúdo, ao ser disponibilizado, não deve se transformar em uma imposição acrítica de informações segmentadas, pois não há aprendizagem sem a participação ativa do aluno no processo. Isolar um dos elementos do processo é problemático, pois ambos são disparadores do processo, e devem ser balanceados ${ }^{15}$.

O estágio extramuros foi a opção mais escolhida pelos entrevistados como estratégia para que os estudantes despertem para a dimensão da relevância social do exercício profissional e para capacitá-los a atuar de forma interdisciplinar em equipe multiprofissional. No segundo tópico para o CD e CE a opção "não há estratégia para isso" foi escolhida com alta frequência. Vale ressaltar que de acordo com a Lei n. $11.788 / 2008^{16}$ que regulamenta os estágios curriculares na graduação, os estágios curriculares devem ser planejados e 
desenvolvidos mediante supervisão docente.

Estudos desenvolvidos ${ }^{17,18}$ conseguiram captar, por meio de avaliações, a tendência de transformação da formação de cirurgiõesdentistas desde a implantação das DCN. Entretanto, permanecem muitas lacunas sobre como as diretrizes podem ser efetivadas nos currículos e práticas educativas ${ }^{17}$.

De forma geral, observa-se que há um longo caminho a ser percorrido pelos cursos de graduação em Odontologia. Na época do estudo, o maior tempo de inscrição de curso no Pró Saúde era de 6 anos, o que deve ser considerado à luz da compreensão de que mudanças profundas requerem tempo de adequação. A decisão política de priorizar a implantação do Pró Saúde ocorreu em virtude da avaliação de que, em muitos cursos da área de Saúde, as DCN, embora retratadas nos projetos pedagógicos, não estavam ainda incorporadas na formatação dos $\operatorname{cursos}^{19}$.

O desafio é transpor os obstáculos para qualificar o ensino no contexto das necessidades de saúde da população, fortalecendo o SUS em todos os níveis de atenção à saúde. Para que essa integração alcance seus objetivos, é preciso que os atores envolvidos assumam de fato seus papeis e atuem fortalecendo cada um dos pilares de sustentação dessa engenhosa estratégia. É preciso que se enxerguem como complementares na formação dos profissionais da odontologia, pois é no dia a dia dos serviços que muitas teorias e discussões realizadas em sala de aula ganham cenários e sujeitos reais ${ }^{20}$.

\section{CONCLUSÕES}

Os cinco cursos incluídos neste estudo de caso demonstraram avanços na reorientação da formação profissional nos cursos de graduação, promovidas e induzidas a partir do advento das DCN e pela implementação do Pró Saúde.

Quanto às estratégias utilizadas podemos dizer que os estágios extramuros apresentam grande variação no seu planejamento, e representam uma ferramenta importante para o desenvolvimento das competências requeridas pelas DCN. Em relação à abordagem pedagógica, ainda predominam práticas tradicionais baseadas na transmissão vertical de conhecimentos e a integração das disciplinas ocorre, principalmente, em seminários modulares e disciplinas profissionalizantes.

\section{ABSTRACT \\ Contribution of Pró-Saúde to a reorientation of Dental education and implementation of the National Curricular Guidelines: case studies}

Pro Health (Pró Saúde) is an initiative of the Ministry of Health in association with the Ministry of Education, with broad coverage and capillarity. It was instituted and implemented since 2006, creating a partnership between health courses with the secretariats of Health, involving, in the beginning, the Brazilian health System (SUS) services and, more recently, involving the other services of SUS, at a national level. The objective of the present study was to verify how and with what strategies the curricular changes are being conducted in undergraduate Dentistry courses at Brazilian public universities enrolled in Pro Health. It is a case study methodology. Five undergraduate courses were selected, one in each macro region of the country, and questionnaires of perception were applied to the coordinators or teaching staff representative of each course, and to a group of students, about the changes in the process. The results pointed out that the Pro Health has been a useful instrument to induce shifts in the direction of the implementation of the National Curricular Guidelines (NCR). The extramural stages present great variation in their planning and represent an important tool for the development of the competencies required by the NCR. Regarding the pedagogical approach, traditional practices based on the vertical transmission of knowledge still predominate, and the integration of the disciplines occurs, mainly, in modular seminars 
and professional disciplines.

Descriptors: Education in Dentistry. Curriculum. Unified Health System. Human Resources in Health.

\section{REFERÊNCIAS}

1. Brasil. Ministério da Educação e Cultura. Conselho Nacional de Educação. Resolução CNE/CES 3/2002.Institui as Diretrizes Curriculares Nacionais do Curso de Graduação em Odontologia. Brasília: Diário Oficial da União, 4 mar 2002. Seção 1, p.10.

2. Brasil. Ministério da Saúde. Programa Nacional de Reorientação da Formação Profissional em Saúde - Pró-Saúde: objetivos, implementação e desenvolvimento potencial. Ministério da Saúde, Ministério da Educação. - Brasília: Ministério da Saúde, 2007. 86 p. [Acesso em 18 jun. 2013]. Disponível em: http://www.prosaude.org/publicacoes/pro saude1.pdf.

3. Secco LG, Pereira MLT. An analysis of the concept of the quality of teaching among course coordinators in the Sao Paulo schools of dentistry. Interface Comun Saúde Educ. 2004;8(15):313-30.

4. Batista MJ, Gibilini C, Kobayashi HM, Ferreira LL, Gonçalo CS, Sousa, MLR. Relato de experiência da interação entre universidade, comunidade e unidade de saúde da família em Piracicaba, SP, Brasil. Arq Odontol. 2010;46(3):144-51.

5. Toassi RFC, Stobäus CD, Mosquera JJM, Moysés SJ. Integrated curriculum for teaching dentistry: new directions for training in the field of healthcare. Interface Comun Saúde Educ. 2012;16(4):529-42.

6. Portaria do Ministério da Saúde ${ }^{\circ}$ 4279/2010) [Acesso em 12 mar. 2017 ]. Disponível em: http://bvsms.saude.gov.br/ bvs/saudelegis/gm/2010/prt4279_30_12_2 010.html.

7. Brasil. Ministério da Saúde. Secretaria de Gestão do Trabalho e da Educação na Saúde. Departamento de Gestão da Educação na Saúde; Brasil. Ministério da Educação. Instituto Nacional de Estudos e Pesquisa. A aderência dos cursos de graduação em Enfermagem, Medicina e Odontologia às Diretrizes Curriculares Nacionais. Brasília; Ministério da Saúde; 2006. 162 p. [Acesso em 18 jun. 2013]. Disponível em: http://bases.bireme.br/cgi) bin/wxislind.exe/iah/online/?IsisScript=ia $\underline{\mathrm{h} / \mathrm{iah} . \mathrm{xis} \& \mathrm{src}=\text { google } \& \text { base }=\text { LILACS } \& \text { la }}$

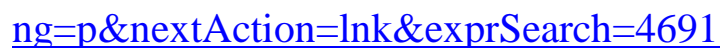
49\&index Search=ID.

8. Lamers JMS, Baumgarten A, Bitencourt FV, Toassi RFC. Mudanças curriculares na educação superior em Odontologia: inovações, resistências e avanços conquistados. Rev ABENO. 2016;16(4):218.

9. Schön DA. Educating the Reflective Practitioner: Toward a New Design for Teaching and Learning in the Professions. San Francisco: Jossey-Bass Publishers 1987: 345p.

10. Moimaz SAS, Wakayama B, Garbin AJI, Garbin CAS, Saliba NA. Análise situacional do estágio curricular supervisionado nos cursos de graduação em Odontologia no Brasil: uma questão de interpretação. Rev ABENO. 2016;16 (4):19-28.

11. Cawahisa PT, Terada RSS, Pascotto RC, Occhi IG, Fujimaki M. Atividades realizadas durante o estágio supervisionado do Curso de Odontologia da UEM em um Centro de Educação Infantil. Cienc Cuid Saude. 2013;12(2) :375-81. 
12. Barbosa FTL, Teixeira SR, Nunes MF, Freire MCM. Implantação das Diretrizes Curriculares Nacionais nos cursos de Odontologia: opinião de formandos de uma universidade pública. Rev ABENO. 2016;16(4):61-71.

13. Anastasiou LGC, Alves LP (Orgs.). Processos de ensinagem na universidade: pressupostos para as estratégias de trabalho em aula. 5.ed. Joinville: UNIVILLE, 2009. $145 p$.

14. BRASIL. MEC. Parâmetros Curriculares Nacionais: Ensino Médio. Brasília: Ministério da Educação 1999: p 89.

15. Klein LR. Fundamentos para uma proposta pedagógica (internet). [Acesso em 11 set. 2015]. Disponível em: http://www.gestao escolar.diaadia.pr.gov.br/arquivos/File/otp /docs_pdf/fundamentos_prop_ped.pdf.

16. Santolini RB. A lei 11.788/08 - A nova lei do estágio. Conteúdo Jurídico, BrasíliaDF. [Acesso em 25 nov. 2015]. Disponível em: http://www.conteudojuridico.c tigo,alei-1178808-a-nova-lei-do-estagio,22752 html.
17. Pessoa TRRF, Noro LRA. Caminhos para a avaliação da formação em Odontologia: construção de modelo lógico e validação de critérios. Ciênc Saúde Colet. 2015;20(7):2277-90.

18. Ribeiro CDM, Maksud I, Koifman L, Alves MGM, Gouvêa MV. O trabalho de campo como dispositivo de ensino, pesquisa e extensão na graduação de Medicina e Odontologia. Interface Comun Saúde Educ. 2013;17(47):947-57.

19. Haddad AE. A enfermagem e a política nacional de formação dos profissionais de saúde para o SUS. Rev Esc Enferm USP. 2011;45(Esp 2):1803-9

20. Faé JM, Silva Jr MF, Carvalho RB, Espoti CDD, Pacheco KTS. A integração ensinoserviço em Odontologia no Brasil. Rev ABENO. 2016; 16(3):7-18

Correspondência para:

Dra Maria Sylvia de Souza Vitalle

e-mail: sylvia.vitalle@gmail.com

Rua Botucatu, 715

04023-062, São Paulo/SP 